\title{
EVALUACIÓN DE LA PRODUCTIVIDAD PRIMARIA DE UN PASTIZAL TEMPLADO EN SANTA FE, ARGENTINA
}

\author{
EVALUATION OF THE PRIMARY PRODUCTIVITY OF TEMPERATE GRASSLAND \\ IN SANTAFE, ARGENTINA
}

\begin{abstract}
Martín, B. ${ }^{1 *}$, Galleano,V. ${ }^{1 * \star}$, Spiller, L.C. ${ }^{1 * \star}$, Vilche, M.S. ${ }^{1}$ y Montico, S. ${ }^{1}$
${ }^{1}$ Facultad de Ciencias Agrarias. Universidad Nacional de Rosario. Zavalla. Santa Fe. Argentina. *bmartin@argentina.com; **ex aequo.
\end{abstract}

\section{Palabras clave adicionales}

Producción estacional. Modelos simples de simulación.

\section{RESUMEN}

El objetivo del trabajo fue generar modelos simples de producción total y estacional de fitomasa aérea de un pastizal templado ubicado en un ambiente de media loma baja. Se utilizaron como predictores precipitaciones, temperaturas medias y relación porcentual entre especies forrajeras, de medición sencilla. El análisis se basó en una serie de ocho años (2002-2009) de datos de producción estacional acumulada de un pastizal ubicado en la localidad de Bustinza, Santa Fe, Argentina. Cada año se estimó la fitomasa aérea por corte manual de 10 muestras de $0,25 \mathrm{~m}^{2}$. El material obtenido se separaró en gramíneas $\mathrm{C}_{3} \mathrm{y}$ $\mathrm{C}_{4}$, anuales y perennes, graminoides y dicotiledóneas y se calculó la composición florística, en base de materia seca. La producción promedio fue de $9051 \mathrm{~kg} / \mathrm{ha}$. Se obtuvieron modelos de regresión satisfactorios entre las precipitaciones anuales y la producción primaria aérea neta; y entre la producción estacional, las precipitaciones estacionales y la relación porcentual entre grupos de especies. Las ecuaciones ajustadas para predecir la producción estacional del pastizal son simples y podrían ser utilizadas en regiones con pastizales de similar estructura.

\section{SUMMARY}

The objective this work was to generate simple models of total and seasonal production for shoot biomass in temperate grassland located in a medium low ridge. Were used as predictors sim-

\author{
AdDITIONAL KEYWORDS \\ Seasonal production. Simple simulation models.
}

ple measurable parameters (rainfall, average temperatures and percentage relationship between forage species). The analysis was based on a series of eight years (2002-2009) of seasonal production data accumulated in a pasture located in Bustinza, Santa Fe, Argentina. In each year the productivity was estimated by manual cutting of 10 samples of $0.25 \mathrm{~m}^{2}$. This material was separated in $\mathrm{C}_{3}$ and $\mathrm{C}_{4}$ grasses, annuals and perennials, graminoids and perennial dicots and estimated the proportion of each fraction in the base of dry matter. The average production was $9051 \mathrm{~kg} / \mathrm{ha}$. The obtained regression models between annual rainfall and net aerial primary production and between production of seasonal rainfall at each station and the percentage ratio between groups of species were satisfactory. The equations to predict the seasonal production of pasture were good and simple estimators, and could be used in other regions having similar grassland structure.

\section{INTRODUCCIÓN}

La región meridional de Santa Fe, Argentina, es una amplia cuenca sedimentaria en donde los bajos gradientes de pendiente constituyen un rasgo general. Abundan las áreas con drenaje deficiente, a menudo ocupando grandes superficies siendo la Pampa Ondulada la subregión geomorfológica mejor drenada de esta gran llanura; 
sin embargo, presenta sectores deprimidos, casi siempre próximos a cursos de agua (Iriondo, 1972; 1987). Allí, las limitaciones de drenaje derivan de la ubicación en el paisaje y de la escasa profundidad de la napa freática (Mosconi et al., 1981). Estos sectores incluyen diferentes suelos y se caracterizan por una conjunción de problemas que afectan la productividad primaria (mal drenaje, anegamientos, salinidad y alcalinidad), determinante en numerosos casos, de una baja aptitud agrícola. Por ello, suelen destinarse a la producción pecuaria en base a pasturas naturales o mejoradas.

La vegetación de los sistemas de producción ubicados en relieve deprimido de la Pampa Ondulada ha sido descripta por diversos autores (Parodi, 1930; Ragonese, 1941; Cabrera y Willink, 1980; Lewis et al., 1985; Lewis, 1995). Fisonómicamente es similar a la vegetación de áreas similares del centro y este de la provincia de Buenos Aires, cuya estructura y funcionamiento fueron ampliamente estudiadas (León, 1975; Sala et al.,1978; León et al., 1979; Hidalgo y Cahuépé, 1991; Lavado et al., 1992; Jacobo et al., 1995; Rodríguez et al., 1997; Rodríguez et al., 1998; Deregibus et al., 2001; Gándara, 2003).

El funcionamiento de las comunidades naturales de la Pampa Ondulada está menos estudiado, pero se han hecho descripciones detalladas de su estructura. Posiblemente la descripción más completa de estos pastizales fue realizada por Lewis et al. (1985) en el SE de Santa Fe. Ellos identificaron tres tipos de comunidades en una cenoclina con restricciones edáficas crecientes, principalmente por saturación hídrica, alcalinidad y salinidad. Desde el borde de los interfluvios hasta las depresiones de los valles de los ríos y arroyos, estas comunidades corresponden a los flechillares, las comunidades halófilas y las comunidades hidrófilas. Estos autores también diferenciaron a los pajonales, como otro agrupamiento caracterizado por su fisonomía y la combinación de salinidad edáfica y anegamientos temporarios.

Las variaciones en la productividad de los pastizales en respuesta a variaciones en las precipitaciones se conocen desde hace mucho tiempo, tanto para los pastizales de la Pampa Deprimida (Soriano, 1991, López et al., 2001) como para los otros en el resto del mundo (Pickup, 1996). En ellos, los incrementos en las lluvias están correlacionados con una mayor producción de biomasa verde y viceversa.

Así, se formula la hipótesis de que la distribución de las precipitaciones y los valores medios de las temperaturas generan cambios en la estructura de la vegetación del pastizal natural, y que estos cambios afectan directamente la productividad primaria del mismo, planteándose como objetivo, la generación de modelos simples de producción total y estacional de fitomasa aérea de un pastizal ubicado en un sector topográficamente previo al deprimido.

\section{MATERIAL Y MÉTODOS}

\section{CARACTERIZACIÓn DEL ÁREA}

El área de estudio está ubicada en las coordenadas geográficas $32^{\prime} 40^{\circ}$ de latitud S y $61^{\prime} 17^{\circ}$ de longitud W. El clima es húmedosubhúmedo mesotermal, con un promedio anual de precipitaciones de $987 \mathrm{~mm}$ y una temperatura media anual de $17^{\circ} \mathrm{C}$ (Estación Meteorológica Zavalla, Servicio Meteorológico Nacional, Argentina; promedios de los 30 años anteriores al período experimental). Entre el 70 y el $75 \%$ de las lluvias ocurren en otoño y primavera, mientras que de diciembre a febrero el balance hídrico medio presenta las mayores deficiencias.

El sitio estudiado está localizado en un sector deprimido, aledaño a una cañada tributaria del arroyo Los Perros (Pampa Ondulada, suroeste de la provincia Santa $\mathrm{Fe})$. El suelo es un Argiudol ácuico, moderadamente bien drenado. El horizonte superficial (A de $19 \mathrm{~cm}$ de profundidad), bien

Archivos de zootecnia vol. 60, núm. 232, p. 966. 
provisto de materia orgánica (2,9\%), es de textura franco-limosa (24\% de arcilla, 70\% de limo y 5,2\% de arena). Los horizontes subsuperficiales: Bt presentan inicialmente textura arcillosa, estructura en prismas simples e irregulares, medios y moderados, con abundantes barnices y moteados, BC tiene estructura franca, escasa cantidad de carbonato en la masa, abundantes barnices y moteados. El horizonte C presenta a partir de los $130 \mathrm{~cm}$ de profundidad una textura franca y sin estructura. El relieve es subnormal con una pendiente promedio de $0,2 \%$.

La vegetación del pastizal está determinada climáticamente, dominando especies de comportamiento fisiológico $\mathrm{C}_{3} \mathrm{y} \mathrm{C}_{4}$. A escala local, la distribución espacial de las comunidades vegetales se relaciona principalmente con la textura del suelo y el régimen hídrico edáfico (Vilche et al., 2008).

\section{DISEÑO DEL MUESTREO Y ANÁLISIS DE LOS DATOS}

El pastizal, de 850 ha, era manejado con pastoreo rotativo, con tiempos de descanso relativamente prolongados. La carga fue de 0,8 - 1 vaca Hereford de $450 \mathrm{~kg}$ de peso vivo/ ha. Se instalaron sobre el mismo 10 sitios de muestreo correspondientes a potreros de 20 ha cada uno, los que fueron seleccionados por presentar similitud en la vegetación y se los consideró como verdaderas repeticiones para las variables estudiadas (Hurlbert, 1994).

Mensualmente se tomó la acumulación de forraje en potreros con descansos de 30, 60 y 90 días. En cada uno de ellos, desde 2002 a 2009, se establecieron aleatoriamente 5 unidades de muestreo de $0,25 \mathrm{~m}^{2}$, donde el fue cortado separando manualmente, las especies presentes y sus valores se promediaron resultando una muestra por sitio. Las fracciones se llevaron a estufa de aire forzado $\left(65^{\circ} \mathrm{C}\right)$ hasta peso constante para determinar el contenido de materia seca (MS).

Con los datos de acumulación de MS, se calcularon las tasas de crecimiento para cada estación. Para evaluar la variación de la composición florística de las muestras, se elaboró una matriz con los valores de MS de las especies presentes en las diferentes submuestras de cada sitio.

Las especies presentes en cada unidad de muestreo se asignaron a distintas categorías de tipos funcionales de plantas: gramíneas anuales y perennes, graminoides y dicotiledóneas, se cuantificó su participación porcentual en el contenido de la materia seca total de la unidad. Además, se agruparon las especies de gramíneas en invernales y estivales de acuerdo a su ciclo de crecimiento $\left(\mathrm{C}_{3} \mathrm{y} \mathrm{C}_{4}\right)$. Se hizo un análisis de clasificación aglomerativo para el agrupamiento de las especies, y la participación porcentual entre ellas en cada estación de crecimiento (RSp). De tal análisis se generaron las siguientes relaciones: en las primaveras las cantidades porcentuales de $\mathrm{C}_{3}$ sumado a la fracción de paspalum respecto al resto de especies presentes; en los veranos las cantidades porcentuales de $\mathrm{C}_{4}$ respecto al resto de las especies presentes en dicha estación; en los otoños las cantidades porcentuales de $\mathrm{C}_{3}$ respecto a las especies $\mathrm{C}_{4} \mathrm{y}$ en los inviernos las cantidades porcentuales de las especies $\mathrm{C}_{3}$ perenne respecto a las $C_{3}$ totales presentes.

Los datos obtenidos se sometieron a análisis de dependencia entre las producciones acumuladas de fitomasa aérea anual y estacional, la RSp normalizada, las precipitaciones acumuladas y las temperaturas medias. Se hicieron regresiones lineales simples y múltiples, previa aplicación del modelo de selección de regresión Stepwise. En todas las pruebas de hipótesis se fijó una tasa de error del 5\% y fue empleado el software estadístico InfoStat (2002).

\section{RESULTADOS}

En el pastizal se encontraron 24 especies dominantes. En la tabla I se presentan aquellas especies que participan en la producción acumulada de forraje y el criterio para 
relacionarlas en cada estación de crecimiento.

\section{VARIABLES CLIMÁTICAS}

Las precipitaciones totales anuales fueron altamente variables comparándolas con los valores históricos (tabla II). El promedio de precipitaciones anuales fue de $872 \mathrm{~mm}$, con un desvío estándar de 308,9 mm, resultando 112,6 mm menos de precipitaciones respecto a la media histórica de la región (1973-2001). Las precipitaciones estacionales promedio tuvieron una distribución bimodal, aunque con gran variabilidad interanual.

Las temperaturas medias siguieron un patrón estacional regular, los valores máximos ocurren en el verano, en tanto que los mínimos en invierno (tabla III). En promedio las temperaturas medias anuales y estacionales, no difirieron de los valores de temperatura histórica.
DESCRIPCIÓN DE LA PRODUCCIÓN DE FITOMASA TOTAL ANUAL

A partir del coeficiente de variación (CV\%) de las producciones totales y estacionales se puede describir la variabilidad entre años (tabla IV).

La máxima producción de fitomasa aérea se registró en el verano, seguida por la primavera, el otoño y fue mínima en el invierno, evidenciando un crecimiento bimodal de la producción anual, con un máximo en el verano (39\% del total), mínimo en el invierno (14\%) y similares para las restantes estaciones climáticas (24 y 23\% para la primavera y otoño, respectivamente).

Se evidenció una importante influencia de las precipitaciones anuales sobre la producción acumulada de la fitomasa aérea del pastizal (figura 1) y una baja asociación con los valores de las temperaturas medias registradas $\left(R^{2}=0,18, n=80\right)$. Es decir, el modelo, cuando fueron consideradas las varia-

Tabla I. Especies presentes en el pastizal (sector topográficamente previo al deprimido de la Provincia de Santa Fe, Argentina) y criterio para relacionarlas en cada estación de crecimiento $(R S p)$. (Species found in grassland (mediun low hill from Santa Fe province, Argentine) and the percentage relation of species evaluated in every station of growth (RSp)).

\begin{tabular}{|c|c|}
\hline Especies dominantes & RSp \\
\hline $\begin{array}{l}\text { C }{ }_{3} \text { Perennes: } \\
\text { Stipa neesiana, Stipa hyalina, Hordeum } \\
\text { stenostachys. } \\
\mathrm{C}_{3} \text { Anual: } \\
\text { Lolium multiflorum, Bromus catharthicus }\end{array}$ & $\begin{array}{l}\text { Primavera } \\
\% \mathrm{C}_{3}+\% \text { pasp / } \% \text { resto de especies }\end{array}$ \\
\hline $\begin{array}{l}\mathrm{C}_{4} \text { Dominante: } \\
\text { Paspalum dilatatum } \\
\text { Otras } \mathrm{C}_{4} \text { : } \\
\text { Panicum bergii, Chloris halophila, Sporobolus } \\
\text { indicus, Cynodon dactylon, etc. }\end{array}$ & $\begin{array}{l}\text { Verano } \\
\% \mathrm{C}_{4} / \% \text { resto de especies }\end{array}$ \\
\hline $\begin{array}{l}\text { Graminoides: } \\
\text { Carex bonariensis, Juncus sp., Cyperus } \\
\text { reflexus }\end{array}$ & $\begin{array}{l}\text { Otoño } \\
\% \mathrm{C}_{3} / \% \mathrm{C}_{4}\end{array}$ \\
\hline $\begin{array}{l}\text { Dicotiledóneas: } \\
\text { Phyla canescens, Cardus acanthoides, } \\
\text { Ambrosia tennifolia, etc. }\end{array}$ & $\begin{array}{l}\text { Invierno } \\
\% \mathrm{C}_{3} \text { perenne } / \% \mathrm{C}_{3} \text { total }\end{array}$ \\
\hline
\end{tabular}

Archivos de zootecnia vol. 60, núm. 232, p. 968. 
Tabla II. Precipitaciones estacionales y totales en los períodos de estudio. (Total and seasonal rainfall in studied periods).

\begin{tabular}{lccccc}
\hline & Prim & Ver & Oto & Inv & Anual \\
\hline $2001 / 02$ & 373,3 & 206,5 & 427,6 & 122,8 & 1130 \\
$2002 / 03$ & 402 & 353 & 349,4 & 100,3 & 1204,7 \\
$2003 / 04$ & 146,6 & 256,5 & 267,6 & 47,8 & 718,5 \\
$2004 / 05$ & 159,7 & 474,4 & 278,4 & 89 & 1001,5 \\
$2005 / 06$ & 332 & 223,5 & 323,7 & 79,2 & 958,4 \\
$2006 / 07$ & 236,7 & 483,3 & 362,7 & 19,3 & 1102 \\
$2007 / 08$ & 75 & 263,8 & 81 & 11,2 & 431 \\
$2008 / 09$ & 233 & 11,8 & 147,1 & 37,8 & 429,75 \\
Promedio & 244,8 & 284 & 279,7 & 63,4 & 872 \\
DES & 116,3 & 153,8 & 115,1 & 40,3 & 308,9 \\
CV (\%) & 48 & 54 & 41 & 64 & 35 \\
PH & 251 & 356 & 283 & 95 & 984,6 \\
\hline
\end{tabular}

PH: Promedio histórico (1973-2001).

bles de manera independiente, según los procedimientos stepwise, mostró que las temperaturas medias no superaban los criterios mínimos para ser incluida, su coeficiente t llevaba asociada una probabilidad crítica superior a 0,05 .

Con la finalidad de encontrar el modelo de ajuste más adecuado para explicar la variabilidad de la producción acumulada en cada estación de crecimiento, se aplicó un análisis univariante para las variables precipitaciones, temperaturas medias y RSp. En este estudio las variables temperatura media y RSp fueron excluidas de las ecuaciones univariantes, detectándose sólo las precipitaciones como variable relevante en algunas estaciones del año (tabla V).

El procedimiento de introducción progresiva stepwise combinó las variables precipitaciones y temperaturas (tabla IV), y posteriormente aceptó un criterio de entrada de las variables regresoras precipitaciones y RSp, excluyendo a la variable temperatura media (tabla V).

\section{DISCUSIÓN}

Los coeficientes de variación de los pro- medios mensuales de las precipitaciones fueron altos, de mayo a septiembre, indicando una importante incertidumbre en la recarga invernal en el perfil del suelo. De octubre a mayo resultaron más estables y con menores coeficientes de variación.

Los valores de la producción total por año obtenidos (tabla IV), fueron diferentes a los encontrados en pastizales similares ubicados en la Cuenca del Salado, los que en clausura presentaron niveles de producción de $3600 \mathrm{~kg} \cdot \mathrm{ha}^{-1}$.año ${ }^{-1}$, durante el primer año, a $5250 \mathrm{~kg} \cdot \mathrm{ha}^{-1}$.año-1 luego de cuatro años (Deregibus y Soriano, 1981), y en pastoreo varían de 5700 (Sala et al.,1981) a 7800 kg.ha ${ }^{-1}$.año-1 (Ansin et al., 2002, 2003) en pastizales de la transición Pampa Deprimida - Pampa Ondulada.

Los pastizales mesotérmicos se conforman con especies de diferentes grupos funcionales $\left(\mathrm{C}_{3} \mathrm{y} \mathrm{C}_{4}\right)$ que contribuyen a generar una curva bimodal (Deregibus, 1988). En este trabajo la producción de fitomasa aérea anual presentó valores de $63 \%$ en la primavera-verano y $37 \%$ otoño-invierno, estos datos son concordantes con ese tipo de curva (tabla I).

Tabla III. Temperaturas medias estacionales y totales en los períodos de estudio. (Total and seasonal temperature in studied periods).

\begin{tabular}{lccccc}
\hline & Prim & Ver & Oto & Inv & Anual \\
\hline $2001 / 02$ & 18,2 & 22,9 & 17,2 & 11,7 & 17,50 \\
$2002 / 03$ & 18,1 & 22 & 16,9 & 11,7 & 17,18 \\
$2003 / 04$ & 18 & 22,5 & 17,2 & 11,5 & 17,30 \\
$2004 / 05$ & 16,6 & 23 & 16,4 & 11,5 & 16,88 \\
$2005 / 06$ & 17,7 & 23,1 & 16,23 & 11,8 & 17,21 \\
$2006 / 07$ & 15,5 & 22,9 & 16,46 & 8,26 & 15,78 \\
$2007 / 08$ & 18,26 & 23,1 & 17,4 & 11,5 & 17,57 \\
$2008 / 09$ & 18,16 & 23,3 & 20,4 & 11,8 & 18,42 \\
Promedio & 17,57 & 22,85 & 17,27 & 11,22 & 17,23 \\
DES & 0,99 & 0,41 & 1,33 & 1,20 & 0,74 \\
CV (\%) & 0,06 & 0,02 & 0,08 & 0,11 & 0,04 \\
PH & 16,93 & 22,90 & 17,16 & 10,76 & 16,94 \\
\hline
\end{tabular}

PH: Promedio histórico (1973-2001). 
Diversos trabajos describen la relación entre la producción de forraje y la precipitación media anual regional (Sala et al., 1988; Lauenroth y Sala, 1992; Echeverría y Molinero, 2000; Oesterheld et al., 2001; Jobbágy y Sala, 2000; Nippert et al., 2006). Los resultados del análisis de la variación interanual de la producción en el pastizal de estudio y las precipitaciones coincidieron con los descriptos por esos autores. Tal respuesta estuvo asociada significativamente $\left(\mathrm{R}^{2}=0,72\right)$ a la influencia que tienen las precipitaciones sobre la productividad del pastizal.

Por otra parte, en pastizales de California, EEUU, con 900 mm de precipitación anual, la productividad se asoció con las precipitaciones anuales, las que tuvieron un efecto directo sobre el rendimiento de las especies que participaban en su estructura, y otro indirecto a través del momento en que se produjo el crecimiento de las especies anuales (Murphy, 1970). En Irlanda se aplicó un modelo de regresión lineal para estimar el rendimiento estacional de una pastura (Han et al., 2003). Se encontró que cuando la disponibilidad de agua no era limitante, la temperatura media podía determinar satisfactoriamente el crecimiento de las especies, sin embargo, esta variable no resultó útil para describir el crecimiento de ese pastizal. En este trabajo, la producción acumulada anual se relacionaría con la capacidad de crecimiento de las especies que componen este pastizal al aumentar las precipitaciones (figura 1).

Al analizar las relaciones entre las precipitaciones y la producción forrajera acumulada estacionalmente se observó que las regresiones no reproducen la magnitud de la tendencia del modelo general (tabla IV y figura 1). Se mejoró el ajuste estacional

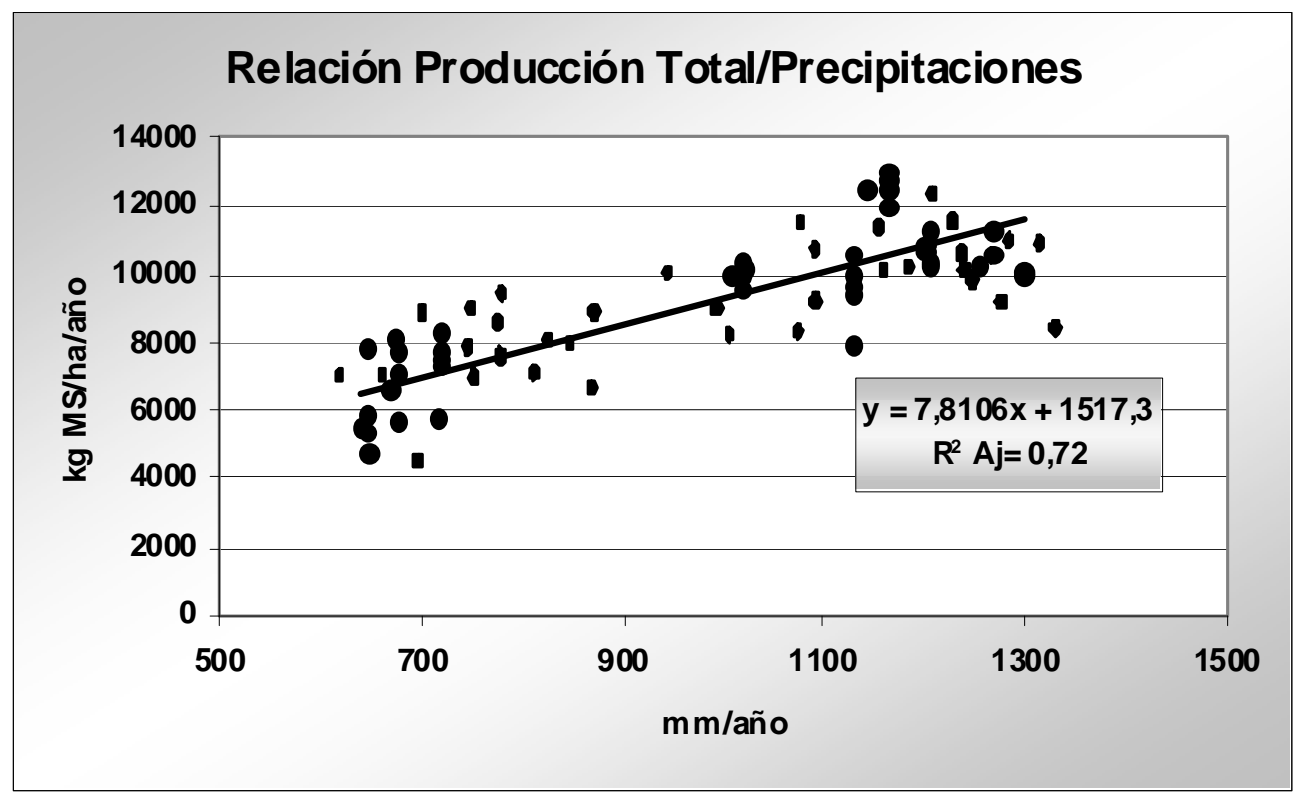

Figura 1. Relación de la producción acumulada total de la fitomasa aérea del pastizal con las precipitaciones anuales $\left(p<0,0001, R^{2} A j=0,72, n=80\right)$. (Grassland net primary production and year rainfall relationship $\left(p<0.0001, R^{2} A j=0.72, n=80\right)$ ). 
sólo en el verano con los valores de las precipitaciones y de las temperaturas, condicionando el nivel de rendimiento de las especies estivales que participaron en el tapiz. Esta dependencia con el crecimiento de las especies fue documentado por otros autores, los que señalan una estrecha relación entre los ciclos de crecimiento de las especies y la variación de las precipitaciones y temperaturas (Fay et al., 2003, Nippert et al., 2006 y Nippert and Knapp, 2007).

Martín(2006), midió en la misma región, tasas diarias de crecimiento de diversas especies de un pastizal similar y estableció que el crecimiento de la mayoría de las especies superaba el 50\%, cuando las precipitaciones eran mayores a la media histórica local en 198 mm. Inversamente, las reducciones en la producción anual o en la producción estacional como consecuencia de las bajas precipitaciones, se explicarían por el escaso contenido de humedad edáfica que podría alterar la actividad radical, el estado hídrico de las especies y su actividad fotosintética. Resultados similares fueron comentados por Fay et al. (2003) para
Tabla IV. Producción de materia seca estacional y total anual del pastizal $\left(\mathrm{kg} \cdot \mathrm{ha}^{-1} . \mathrm{tiempo}^{-1}\right.$ ) y porcentaje de la producción primaria estacional respecto al valor total (\%/PTA). (Seasonal total grassland dry matter production $(\mathrm{kg} / \mathrm{ha} / \mathrm{time})$ and relation between seasonal and total primary production (\%/PTA)).

\begin{tabular}{lccccr}
\hline & Prim & Ver & Oto & Inv & PTA \\
\hline $2001 / 02$ & 2675 & 1626 & 2646 & 1393 & 8340 \\
$2002 / 03$ & 3158 & 3728 & 2461 & 1879 & 11226 \\
$2003 / 04$ & 1283 & 1944 & 1833 & 967 & 6027 \\
$2004 / 05$ & 1128 & 3867 & 1983 & 1621 & 8599 \\
$2005 / 06$ & 2567 & 4861 & 3705 & 1513 & 12646 \\
$2006 / 07$ & 2816 & 3634 & 1962 & 1531 & 9943 \\
$2007 / 08$ & 2085 & 4593 & 1343 & 576 & 8597 \\
$2008 / 09$ & 1398 & 4286 & 451 & 893 & 7028 \\
Promedio & 2139 & 3567 & 2048 & 1297 & 9051 \\
DES & 781,5 & 1181,2 & 954,6 & 438,5 & 2158 \\
CV (\%) & 37 & 33 & 47 & 34 & 24 \\
$\%$ de PTA & 24 & 39 & 23 & 14 & \\
\hline
\end{tabular}

PTA: Producción total.año-1.

pastizales dominados por especies $\mathrm{C}_{4}$, Heitschmidt y Vermeire (2006) para pasti-

Tabla $\mathbf{V}$. Relación de la producción estacional de fitomasa aérea, precipitaciones acumuladas y temperaturas medias, para esas estaciones climáticas. (Seasonal grassland production and rainfall relationship and mean temperatures relationship for those seasons).

a) $y=a \pm b_{x}(y=$ producción acumulada; $x=$ precipitaciones). b) $y=a \pm b_{0} x_{0} \pm b_{1} x_{1}\left(y=\right.$ producción acumulada; $x_{0}=$ precipitaciones; $x_{1}=$ temperaturas medias).

\begin{tabular}{lcccccccccc}
\hline & $\mathrm{a}$ & $\mathrm{b}$ & $\mathrm{R}^{2} \mathrm{Aj}$ & $\mathrm{p}$ & & $\mathrm{a}$ & $\mathrm{b}_{0}$ & $\mathrm{~b}_{1}$ & $\mathrm{R}^{2} \mathrm{Aj}$ & $\mathrm{p}$ \\
\hline Primavera & 633,4 & 5,97 & 0,56 & 0,0001 & Primavera & 56,41 & 5,99 & 33,27 & 0,55 & 0,0001 \\
Verano & 20,72 & 9,45 & 0,59 & 0.0001 & Verano & -17737 & 9,43 & 776 & 0,68 & 0,0001 \\
Otoño & 587,6 & 5,1 & 0,30 & 0,0003 & Otoño & 8574 & 2,23 & $-416,7$ & 0,52 & 0,0001 \\
Invierno & - & - & NS & NS & Invierno & - & - & - & NS & NS
\end{tabular}

a: constante y representa el valor que se asigna a la producción (y) en el caso de que las precipitaciones $(x)$ fueran igual a 0 ;

b: incremento de la producción acumulada, en promedio, por cada $\mathrm{mm}$ de aumento en las precipitaciones;

$R^{2} A j ; p<0,05 ; n=40 ; N S$ : No significativo. a: valor que se asigna a la producción acumulada (y) en el caso de que las precipitaciones $\left(x_{0}\right)$ y temperaturas medias $\left(x_{1}\right)$ fueran igual a 0 ;

b: incremento de la producción acumulada, en promedio, por cada $\mathrm{mm}$ de aumento en las precipitaciones $\mathrm{y}^{\circ} \mathrm{C}$ de aumento en las $\mathrm{T}^{\circ}$ medias; $R^{2} A j ; p<0,05 ; n=40 ; N S$ : No significativo. 
zales de zonas con $340 \mathrm{~mm}$ y en aquellos con $1483 \mathrm{~mm}$ de precipitaciones anuales (Garbulsky, 2004). En este estudio, los aumentos lineales de la producción acumulada en primavera, verano y en otoño mostrarían que existe una mayor capacidad de producción de materia seca de algunas especies dominantes de este pastizal y en esas estaciones.

En el periodo invernal, la relación entre producción, precipitaciones y temperaturas no fue significativa. Esta aparente estabilidad en la producción se asociaría a la presencia de gramíneas $\mathrm{C}_{3}$ anuales y perennes. Ambos grupos manifiestan las máximas tasas de crecimiento de la materia seca de una manera opuesta a las condiciones ambientales de sequía o de exceso de precipitaciones invernales. Martín (2006) determinó tasas de crecimiento diarias desigua-

Tabla VI. Regresiones lineales múltiples entre la producción acumulada de fitomasa aérea $\left(\mathrm{kg} \mathrm{ha}^{-1}\right.$.período $\left.{ }^{-1}\right)$ como variable regresora y las precipitaciones $\left(x_{0}\right.$, mm.período ${ }^{-1}$ ) y el aporte porcentual de las especies dominantes para cada estación climática $\left(x_{1}\right)$, según la ecuación $y=a \pm$ $b_{0} x_{0} \pm b_{1} x_{1}, n=40$. (Linear multiple regressions between production accumulated of biomass $(\mathrm{kg}$ ha/period) as repressor variable and the rainfalls $\left(\mathrm{x}_{0}, \mathrm{~mm} /\right.$ period) and the percentage contribution of the dominant species for every climatic station $\left(x_{1}\right)$, according to equation $\left.y=a \pm b_{0} x_{0} \pm b_{1} x_{1}, n=40\right)$.

\begin{tabular}{lcccccc}
\hline & $a$ & $b_{0}$ & $b_{1}$ & $R^{2} A j$ & $\begin{array}{c}p \\
\text { (modelo) }\end{array}$ \\
\hline Primavera & $-2102,4$ & 6,48 & 41,34 & 0,87 & $<0,0001$ \\
Verano & 539,31 & 3,5 & 28,22 & 0,71 & $<0,0001$ \\
Otoño & $-640,6$ & 7,16 & 111,4 & 0,73 & $<0,0001$ \\
Invierno & 1698,6 & 0,21 & $-6,04$ & 0,36 & $<0,0006$
\end{tabular}

$a$ : constante; $b_{0}$ pendiente de la recta de la variable Iluvias acumuladas; $b_{1}$ pendiente de la recta para la variable $\% \mathrm{C}_{3}+$ pasp/resto de especies (para primavera); \% $\mathrm{C}_{4} /$ resto de especies (para el verano); \% $\mathrm{C}_{3} / \mathrm{C}_{4}$ (para el otoño) y $\% \mathrm{C}_{3}$ perenne/C $\mathrm{C}_{3}$ total (para el invierno). les entre $L$. multiplorum y $H$. stenostachis ante determinadas situaciones de temperatura y humedad edáfica, condición que lograría complementar los crecimientos y mantener relativamente constante la producción de cada uno de los inviernos evaluados.

Los trabajos de Walker (1992), Sala et al. (1986) y Ferrer et al. (2001) señalan que es necesario agrupar a las especies que componen un pastizal en características funcionales de crecimiento, para poder predecir la productividad del mismo y establecer comparaciones entre pastizales similares. Por su parte Lacorte y Goldfarb (1996) estudiando durante 5 años el comportamiento de un pastizal a cielo abierto en el sur de la provincia de Misiones, Argentina, determinaron la estrecha correlación entre precipitaciones y productividad primavero-estival. Los autores atribuyeron la magnitud de los valores de producción a la variación en la composición botánica, sin incluir su ponderación en dichos modelos predictivos. Así, la clasificación aplicada en este trabajo, en base a las tasas de crecimiento de las especies que lo componen en cada estación de crecimiento (Martín, 2006), permitió ajustar significativamente las producciones de materia seca con los valores de las precipitaciones ocurridas en las estaciones de crecimiento primavero-estivo-otoñal (tabla VI).

Las producciones invernales tuvieron, en general, pocas variaciones frente a los diversos registros de precipitaciones ocurridas en el período de estudio (2002/2009). Se logró mejorar la predicción $\left(\mathrm{R}^{2}=0,36\right)$ cuando se agregó la relación porcentual entre especies de crecimiento $\mathrm{C}_{3}$ perennes (H. stenostachis y S. hyalina y S. neesiana) y las $\mathrm{C}_{3}$ anuales (L. multiflorum). Wardle (2001) asevera que no es la diversidad per se sino los rasgos funcionales de las especies dominantes y sus relaciones los que controlan fundamentalmente el funcionamiento del pastizal, concepto aplicable en esta estación de crecimiento.

Luego de ocho años de observación, se

Archivos de zootecnia vol. 60, núm. 232, p. 972. 
logró establecer ajustes de las regresiones que indican tendencias reales de la producción forrajera en estos tipos de pastizales.

\section{CONCLUSIONES}

Las precipitaciones anuales influyen en la disponibilidad de fitomasa aérea de los pastizales templados del sur de Santa Fe y las estivales son las que poseen mayor capacidad para determinar la productividad primaria.

La estacionalidad de la producción está mayormente descripta por las precipitaciones y la relación entre el aporte porcentual de especies dominantes presentes.

Las relaciones halladas entre la producción estacional del pastizal y las variables climáticas, constituyen una primera aproximación al reconocimiento de la dinámica

\section{BIBLIOGRAFÍA}

Ansín, O.E., Oyhamburu, E.M., Vecchio, M.C., Eirin, M.A. y Cordero, M.I. 2002. Composición botánica y condición forrajera de un pastizal de media loma en diferentes manejos de pastoreo. Rev. Arg. Prod. Anim., 22 (Supl. 1): 209-210.

Ansín, O.E., Oyhamburu, E.M., Vecchio, M.C., Heguy, B., Cordero, M.I. y Eirin, M.A. 2003. Efecto del manejo del pastoreo sobre la biomasa neta de forraje acumulada en dos tipos de pastizales templados de la Pampa Deprimida. Rev. Arg. Prod. Anim., 23 (Supl.1): 151-152.

Cabrera, A.L y Willink, A. 1980. Biogeografía de América Latina. Serie Biología. Monografía 13. $2^{a}$ Edición. Programa Regional de Desarrollo Científico y Tecnológico. Organización de Estados Americanos. Washington. DC. 122 pp.

Deregibus, V.A. y Soriano, A. 1981. Los pastizales de la zona de cría de la Depresión del Salado desde el punto de vista ecológico. Rev. Arg. Prod. Anim., 1: 60-83.

Deregibus, V.A. 1988. Importancia de los pastizales naturales en la República Argentina: Situación presente y futura. Rev. Arg. Prod. Anim., 8: 6776.

Deregibus, V.A., Jacobo, E. and Ansin, O.E. 2001. Grassland use and plant diversity in grazed estacional de la productividad de los pastizales templados locales, en situaciones paisajísticas asociadas a sectores perideprimidos. La misma está íntimamente relacionada con las especies participantes, la importancia relativa de aquellas dominantes y las precipitaciones que ocurrieron en cada estación climática. La relevancia de este abordaje radica en la disminución de la incertidumbre inherente a la complejidad de los sistemas de producción y a la cuantificación, aun parcial, de estos procesos.

\section{AGRADECIMIENTOS}

Los autores agradecen la colaboración prestada al Ing. Agr. Lelio Gasparotti, personal a cargo de la Estancia "La Patria", al Sr. Andrés von Buch y al CREA San Jorge las Rosas, Santa Fe, Argentina.

ecosystems. Proceeding of the XIX International Grassland Congress. Brazil. pp. 879-882.

Echeverría, J.C. y Molinero, H.B. 2000. Modelo de evaluación de pastizales en san Luis. VIII Jornada Modelo Ambiental (CNM). U.N.S.L. San Luis. Argentina. 112 pp.

Fay, P.A., Carlisle, J.D., Knapp, A.K., Blair, J.M. and Collins, S.L. 2003. Productivity responses to altered rainfall patterns in a C4-dominanted grassland. Oecologia, 137: 245-251.

Ferrer, C., Barrantes, O. y Broca, A. 2001. La noción de biodiversidad en los ecosistemas pascícolas españoles. Pastos, 31: 129-184.

Gándara, F. 2003. Manejo del campo natural. INTA EEA Colonia Benítez. Argentina. Hoja Informativa. 5 pp.

Garbulsky, M.F. 2004. Distribución y funcionamiento de las áreas protegidas de Argentina. Diferencias funcionales con áreas bajo uso agropecuario. Tesis de Magister. Universidad de Buenos Aires. Argentina. http://www. scielo.org/php/reference.php (09/10/10).

Han, D., Kiely P.O. and Sun, D.W. 2003. Application of water-stress models to estimate the herbage dry matter yield of a permanent grassland pasture sward regrowth. Biosyst. Engin., 84: 101-111. 


\section{MARTÍN, GALLEANO, SPILLER, VILCHE Y MONTICO}

Heitschmidt, R.K. and Vermiere, L.T. 2006. Can abundant summer precipitation counter losses in herbage production be caused by spring drought?. Rang. Ecol. Manage., 59: 392-399.

Hidalgo, L. y Cauhepé, M.A. 1991. Producción de forraje de las comunidades forrajeras de la Pampa Deprimida. Rev. CREA, 145: 58-64.

Hurlbert, S.H. 1994. Old shibboleths and new syntheses. Review of design and analysis of ecological experiments. S.M. Scheiner and J. Gurevitch (Eds.). Trends Ecol. Evol., 9: 495496.

Iriondo, M.H. 1972. Mapa geomorfológico de la llanura aluvial del río Paraná desde Helvecia hasta San Nicolás. República Argentina. Rev. Asoc. Geol. Argentina, 27: 155-160.

Iriondo, M.H. 1987. Geomorfología y cuaternario de la provincia de Santa Fe. Corrientes. Argentina. Rev. D'Orbignyana, 4: 1-54.

Jacobo, E., Rodriguez, A., Garbulsky, M., Agnusdei, M., Refi, R. y Deregibus, V. 1995. Cambios estructurales generados por el pastoreo controlado en pastizales de la Pampa Deprimida. XVII Reunión Argentina de Ecología. Córdoba. Argentina. 182 pp.

Jobbágy, E.G. and Sala, O.E. 2000. Controls of grass and shrub aboveground production in the Patagonian steppe. Ecol. Applic., 10: 541-549

Lacorte, S.M. y Goldfarb, M.C. 1996. Evaluación agronómica de un pastizal nativo del sur de Misiones. Miscelánea No 34. INTA EEA Cerro Azul. 16 pp.

Lauenroth, W.K. and Sala, O.E. 1992. Long-term forage production of North American shortgrass steppe. Ecol. Applic., 2: 397-403.

Lavado, R., Rubio, G. and Alconada, M. 1992. Grazing management and soil salinitation in two Pampean natraqualfs. Turrialba, 42: 500-508.

León, R.J.C. 1975. Las comunidades herbáceas de la región de Castelli-Pila. La Plata. Comisión de Investigaciones. Argentina. Monografía 5: 73-107.

León, R.J.C., Burkart, S. y Movia, C. 1979. Relevamiento fitosociológico del pastizal del norte de la Depresión del Salado. INTA. Serie Fitogeografica. $n^{\circ}$ 17. 94 pp.

Lewis, J.P., Collantes, M., Pire, E., Carnevale, N., Bocanelli, S., Stofella, S. and Prado, D. 1985. Floristic groups and plant communities of southeastern Santa Fe. Argentina. Vegetatio,
60: 67-90.

Lewis, J.P. 1995. Pastizales y sabanas de la provincia de Santa Fe. Argentina. En: Pastizales y sabanas de América del Sur. CYTEC-CIELAT. cap 5: 77-100.

López, M.V., Arias Mañotti, A.A., Pace, G.J., Casco, J.F., Goldfarb, M.C. y Giménez, L. 2001. Programa para simular el rendimiento de materia seca de pastizales de la región noroeste de la provincia de Corrientes mediante variables climáticas. Rev. Arg. Prod. Anim., 21: 88-89.

Martín, B. 2006. Producción primaria y calidad forrajera de un pastizal pampeano y su reemplazo por pasturas. (Tesis MSc). Universidad Nacional de Rosario. UNR. Argentina. T No 258. Biblioteca Facultad Ciencias Agrarias. 150 pp. http://www.fcagr.unr.edu.ar/ (13/01/09).

Mosconi, F., Priano, J., Hein, N., Moscatelli, G., Salazar, J., Gutiérrez, T. y Cáceres, L. 1981. Mapa de suelos de la provincia de Santa Fe. Tomo I. Ed. INTA-MAG. Santa Fe. Argentina. 248 pp.

Murphy, A. H. 1970. Predicted forage yields based on fall precipitation in California annual grasslands. J. Range Manage., 23: 363-365.

Nippert, J.B., A.K. Knapp and J.M. Briggs. 2006. Intra-annual rainfall variability and grassland productivity: can the past predict the future? Plant Ecol., 184: 65-74.

Nippert, J.B. and A.K. Knapp. 2007. Linking water uptake with rooting patterns in grassland species. Oecologia, 153: 261-272.

Oesterheld, M, Loreti, J., Semmartin, M. and Sala, O.E. 2001. Interannual variation in primary production of a semi-arid grassland related to previous year production. J. Veg. Sci., 12: 137-142.

Parodi, L. 1930. Ensayo fitogeográfico sobre el partido de Pergamino. Argentina. Rev. Fac. Agron. Vet., 7: 65-271.

Pickup, G. 1996. Estimating the effects of land degradation and rainfall variation on productivity in rangelands: an aproach using remote sensing and models of grazing and herbage dynamics. J. Appl. Ecol., 33: 819-832.

Ragonese, A. E. 1941. La vegetación de la provincia de Santa Fe. Darwiniana, 5: 369-415.

Rodriguez, A., Jacobo, E., Garbulsky, M. y Deregibus, V. 1997. Efecto del pastoreo y las precipitaciones sobre la estructura del pastizal de la Pampa Deprimida. Rev. Arg. Prod. Anim., 


\section{EVALUACIÓN DE LA PRODUCTIVIDAD PRIMARIA DE UN PASTIZAL TEMPLADO}

17 (Supl. 1): 156-157.

Rodriguez, A., Jacobo, E y Deregibus, V. 1998. Modalidades de pastoreo: su impacto sobre la morfología de algunas especies clave del pastizal de la Pampa Deprimida. Rev. Arg. Prod. Anim., 18 (Supl. 1): 148-149.

Sala, O.E., Deregibus, V.A., Schlichter, T. y Alippe, H. A. 1978. Productividad primaria neta aérea de un pastizal de la Depresión del Salado. Comisión de Investigaciones Científicas. La Plata. Argentina. Monografía 8: 4-38.

Sala, O.E., Deregibus, V.A., Schlichter, T. and Alippe, H.A. 1981. Productivity dynamics of a native temperate grassland in Argentina. $J$. Range Manage., 34: 48-51.

Sala, O.E., Oesterheld, M., León, R.J.C. and Soriano, A. 1986. Grazing effects upon plant community structure in subhumid grasslands of Argentina. Vegetatio, 67: 27-32.
Sala, O.E., Parton, W.J., Joyce, L.A. and Lauenroth, W.K. 1988. Primary production on the central grassland region of United States. Ecology, 69: 40-45.

Soriano, A. 1991. Rio De Plata Grasslands. In: Ecosystems of the world: Natural grasslands. Vol. 8A. Elsevier. Amsterdam. pp. 367-408.

Vilche, M.S., Martín, B., Spiller, L.C. y Galleano, A. 2008. Interrelación suelo-vegetación en un pastizal natural del centro-oeste de Santa Fe. En: Actas X Congreso y XVIII Reunión Anual Sociedad de Biología. Rosario. Santa fe. Argentina. pp. 44-45.

Walker, B.H. 1992. Biodiversity and ecological redundancy. Cons. Biol., 6: 18-23.

Wardle, D.A. 2001. Experimental demonstration that plant diversity reduce invisibility evidence of a biological mechanism or a consequence of sampling effect?. Oikos, 95: 161-170. 